\title{
Existence of local degrees of freedom for higher dimensional pure Chern-Simons theories
}

\author{
Máximo Bañados \\ Centro de Estudios Cientificos de Santiago, Casilla 16443, Santiago, Chile \\ and Theoretical Physics Group, The Blackett Laboratory, Imperial College, London SW7 2BZ, United Kingdom \\ Luis J. Garay \\ Theoretical Physics Group, The Blackett Laboratory, Imperial College, London SW7 2BZ, United Kingdom \\ Marc Henneaux \\ Centro de Estudios Científicos de Santiago, Casilla 16443, Santiago, Chile \\ and Université Libre de Bruxelles, Campus Plaine, C.P. 231, B-1050, Bruxelles, Belgium
}

(Received 28 June 1995)

\begin{abstract}
The canonical structure of higher dimensional pure Chern-Simons theories is analyzed. It is shown that these theories have generically a nonvanishing number of local degrees of freedom, even though they are obtained by means of a topological construction. This number of local degrees of freedom is computed as a function of the spacetime dimension and the dimension of the gauge group.
\end{abstract}

PACS number(s): 11.10.Kk, 11.15.Tk

Three-dimensional pure Chern-Simons theory is well known to possess higher dimensional generalizations. These generalizations are theories in $2 n+1$ dimensions constructed from characteristic classes in $2 n+2$ dimensions in exactly the same way as three-dimensional Chern-Simons theory is built out of the four-dimensional characteristic classes. More precisely, if $F^{a}$ is the curvature two-form $F^{a}=d A^{a}$ $+\frac{1}{2} f_{b c}^{a} A^{b} \wedge A^{c}$ associated with the gauge field one-form $A^{a}$, where $f_{b c}^{a}$ are the structure constants of the gauge group, and $g_{a_{1} \cdots a_{n+1}}$ is a rank $n+1$, symmetric tensor invariant under the adjoint action of the gauge group, then one defines the Chern-Simons Lagrangian $\mathscr{L}_{\mathrm{CS}}^{2 n+1}$ through the formula

$$
d \mathscr{D}_{\mathrm{CS}}^{2 n+1}=g_{a_{1} \cdots a_{n+1}} F^{a_{1}} \wedge \cdots \wedge F^{a_{n+1}} .
$$

The three-dimensional case is obtained by taking $n=1$, which yields $d \mathscr{L}_{\mathrm{CS}}^{3}=g_{a b} F^{a} \wedge F^{b}$, where $g_{a b}$ is an invariant metric on the Lie algebra (necessarily proportional to the Killing metric if the Lie algebra is simple).

The Chern-Simons action $I=\int_{M} \mathscr{C}_{\mathrm{CS}}^{2 n+1}$ is invariant under standard gauge transformations

$$
\delta_{\epsilon} A_{\mu}^{a}=D_{\mu} \epsilon^{a}
$$

It is also invariant under spacetime diffeomorphisms, $\delta_{\eta} A_{\mu}^{a}=£_{\eta} A_{\mu}^{a}$, since $\mathscr{L}_{\mathrm{CS}}^{2 n+1}$ is a $(2 n+1)$-form. The spacetime diffeomorphisms can also be represented as

$$
\delta_{\eta} A_{\mu}^{a}=\eta^{\nu} F_{\mu \nu}^{a}
$$

Indeed, these symmetries differ from the Lie derivative only by a gauge transformation and are often called improved diffeomorphisms [1]. If the only symmetries of the ChernSimons action are the diffeomorphisms (3) and the gauge transformations (2), then we shall say that there is no accidental gauge symmetry. How this translates into an algebraic condition on $g_{a_{1} \cdots a_{n+1}}$ will be described precisely below.
Of particular interest are the Chern-Simons theories with gauge group $\mathrm{SO}(2 n+1,1)$ or $\mathrm{SO}(2 n, 2)$ in $2 n+1$ dimensions because they define gravitational theories [2]. For $n=1$, one recovers the standard Chern-Simons formulation of Einstein gravity with a cosmological constant [3]. For $n>1$ one gets the Einstein-Hilbert action supplemented by Lovelock terms [4] with definite coefficients. These gravitational theories admit intriguing black hole solutions [5] generalizing the three-dimensional black holes of Ref. [6].

One of the striking features of Chern-Simons theory in three dimensions is the fact that it has no local degrees of freedom. This is because the equations of motion

$$
g_{a a_{1} \cdots a_{n}} F^{a_{1}} \wedge \ldots \wedge F^{a_{n}=0}
$$

reduce to $F^{a}=0$ in the three-dimensional case. Thus, the space of solutions of Chern-Simons theory in three dimensions is the finite-dimensional moduli space of flat connections modulo gauge transformations. (Note that the diffeomorphisms lead to no further quotientizing because they vanish on shell.)

Since the higher dimensional Chern-Simons theories are constructed along the same topological pattern as their threedimensional analogue, one may wonder whether they are also devoid of local excitations and have only global degrees of freedom. One of the purposes of this Rapid Communication is to explain why this is not the case. We also count explicitly the number of local degrees of freedom as a function of the dimensions of spacetime and of the gauge group. It turns out that the crucial ingredient that controls the whole analysis is the invariant tensor $g_{a_{1} \cdots a_{n+1}}$.

We start the discussion with the five-dimensional case $(n=2)$ and an $N$-dimensional Abelian group $\left[G=\mathrm{U}(1)^{N}\right]$. This case already contains all the main points that we want to address and is particularly simple because the invariance condition imposes no restriction on the tensor $g_{a_{1}} \cdots a_{n+1}$. We shall deal with the general situation of an arbitrary gauge group afterward. 
Assume first that there is only one single Abelian field. The equations of motion imply $F \wedge F=0$; i.e., $F$ has at most rank 2 . In the generic case, $F$ has exactly rank 2 (in the space of solutions of $F \wedge F=0$, the solution $F=0$ has measure zero). Since $F$ is a closed two-form, one may bring it locally to the canonical form $F=d x^{1} \wedge d x^{2}$ by a diffeomorphism (Darboux theorem for presymplectic forms of rank 2). Thus, the quotient space of the solutions of the equations of motion modulo the gauge transformations (2) and spacetime diffeomorphisms (3) has locally one and only one solution. This implies that the theory has no local degrees of freedom, in agreement with the findings of Ref. [7].

The case of a single Abelian gauge field is, however, a poor representative of what happens in the general situation and, in that sense, is somewhat misleading. The reason is that, in contrast with the three-dimensional Chern-Simons theory, we have also used the diffeomorphisms to prove the absence of local degrees of freedom. Indeed, these diffeomorphisms are needed to bring $F$ to its canonical form. But if there are many Abelian fields, then there are many $F$ 's to be brought simultaneously to canonical form and this is not possible with a diffeomorphism. Thus, for many $(N>1)$ Abelian fields, one expects the existence of local degrees of freedom unless the invariant tensor $g_{a b c}$ happens to have been chosen in some peculiar way that enlarges the number of gauge symmetries of the theory (accidental gauge symmetries).

A typical example of a theory with accidental gauge symmetries is obtained by taking all the mixed components of $g_{a b c}$ to vanish, so that the action is just the direct sum of $N$ copies of the action for a single Abelian field. The theory is then clearly invariant under diffeomorphisms acting independently on each copy and has no degrees of freedom. But there is no reason to take vanishing mixed components for $g_{a b c}$. If these mixed components differ from zero (and cannot be brought to zero by a change of basis), then the action is not invariant under diffeomorphisms acting independently on each gauge field component $A^{a}$, because the invariance of the cross terms requires the diffeomorphism parameters for each copy to be equal, thus gluing all of them together in a single symmetry.

In order to substantiate this discussion, let us count precisely the number of local degrees of freedom. This number does not depend on which method (Lagrangian or Hamiltonian) one chooses to compute it [8]. In our case, it turns out to be more convenient to follow the Hamiltonian analysis [9]. To that end we shall assume that the spacetime manifold $M$ has the topology $\mathfrak{R} \times \Sigma$, where $\Sigma$ is a four-dimensional manifold. We then decompose the spacetime gauge field oneform $A^{a}$ as $A_{\mu}^{a} d x^{\mu}=A_{0}^{a} d t+A_{i}^{a} d x^{i}$ where the coordinate $t$ runs over $\mathfrak{R}$ and the $x^{i}$ are coordinates on $\Sigma$. Although there is no spacetime metric to give any meaning to expressions such as timelike or spacelike, we will call time the coordinate $t$ and we will say that $\Sigma$ is a spacelike section as shorthand expressions.

It is easy to see that the Chern-Simons action depends linearly on the time derivative of $A_{i}^{a}$,

$$
I=\int_{\Re} \int_{\Sigma}\left[l_{a}^{i}\left(A_{j}^{b}\right) \dot{A}_{i}^{a}-A_{0}^{a} K_{a}\right]
$$

where $K_{a}$ is given by

$$
K_{a}=-g_{a b c} \epsilon^{i j k l} F_{i j}^{b} F_{k l}^{c} .
$$

The explicit form of the function $l_{a}^{i}\left(A_{j}^{b}\right)$ appearing in Eq. (5) is not needed here but only its "exterior" derivative in the space of spatial connections, which reads

$$
\Omega_{a b}^{i j} \equiv \frac{\delta l_{b}^{j}}{\delta A_{i}^{a}}-\frac{\delta l_{a}^{i}}{\delta A_{j}^{b}}=-4 \epsilon^{i j k l} g_{a b c} F_{k l}^{c} .
$$

The equations of motion obtained by varying the action with respect to $A_{i}^{a}$ are given by

$$
\Omega_{a b}^{i j} \dot{A}_{j}^{b}=\Omega_{a b}^{i j} D_{j} A_{0}^{b},
$$

while the variation of the action with respect to $A_{0}^{a}$ yields the constraint $K_{a}=0$.

Since the action is linear in the time derivatives of $A_{i}^{a}$, the canonically conjugate momenta $p_{a}^{i}$ are subject to the $4 N$ primary constraints

$$
\phi_{a}^{i}=p_{a}^{i}-l_{a}^{i} \approx 0 .
$$

These constraints transform in the coadjoint representation of the Lie algebra because the inhomogeneous terms in the transformation laws of $p_{a}^{i}$ and $l_{a}^{i}$ cancel out.

It turns out to be more convenient to replace the constraints $K_{a}$ by the equivalent set

$$
G_{a}=K_{a}-D_{i} \phi_{a}^{i}
$$

The surface defined by $K_{a}=0, \phi_{i}^{a}=0$ is equivalent to the surface defined by $G_{a}=0, \phi_{i}^{a}=0$. The new constraints $G_{a}$ generate the gauge transformations (2), e.g., $\left\{A_{i}^{a}, \int_{\Sigma} \lambda^{b} G_{b}\right\}=D_{i} \lambda^{a}$.

The Hamiltonian action takes the form [9]

$$
I=\int_{\mathfrak{R}} \int_{\Sigma}\left[p_{a}^{i} \dot{A}_{i}^{a}-A_{0}^{a} G_{a}-u_{i}^{a} \phi_{a}^{i}\right]
$$

The Poisson bracket among the constraints is given by

$$
\begin{gathered}
\left\{\phi_{a}^{i}, \phi_{b}^{j}\right\}=\Omega_{a b}^{i j}, \\
\left\{\phi_{a}^{i}, G_{b}\right\}=f_{a b}^{c} \phi_{c}^{i}, \\
\left\{G_{a}, G_{b}\right\}=f_{a b}^{c} G_{c},
\end{gathered}
$$

where $f_{a b}^{c}$ are the structure constants of the Lie algebra, which vanish in the Abelian case that we are considering now. It follows from the constraint algebra that there are no further constraints. The consistency condition $\dot{G}_{a}=0$ is automatically satisfied because $G_{a}$ is first class while the other consistency equation $\dot{\phi}_{a}^{i}=\Omega_{a b}^{i j} u_{j}^{b}=0$ will just restrict some of the Lagrange multipliers $u_{j}^{b}$.

Equations (13) and (14) reflect that the constraints $G_{a}$ are the generators of the gauge transformations and that the constraints $\phi_{a}^{i}$ transform in the coadjoint representation. This means, in particular, that the $G_{a}$ 's are first class, as mentioned above. 
The nature of the constraints $\phi_{a}^{i}$ is determined by the eigenvalues of the matrix $\Omega_{a b}^{i j}$. It turns out that the matrix $\Omega_{a b}^{i j}$ is not invertible on the constraint surface $K_{a}=0$. Indeed, using some simple combinatorial identities, one can prove that $K_{a}$ given in Eq. (6) and $\Omega_{a b}^{i j}$ satisfy the relation

$$
\Omega_{a b}^{i j} F_{k j}^{b}=\delta_{k}^{i} K_{a} .
$$

This equation shows that, on the constraint surface $K_{a}=0$, the matrix $\Omega_{a b}^{i j}$ has four null eigenvectors $\left(v_{k}\right)_{j}^{b}=F_{k j}^{b}$, $(k=1, \ldots, 2 n)$. The corresponding four first-class constraints, namely,

$$
H_{i} \equiv F_{i j}^{a} \phi_{a}^{j},
$$

generate the spatial diffeomorphisms (3). They satisfy the spatial diffeomorphism algebra, up to gauge transformations. The presence of these constraints is of course not a surprise because the Chern-Simons action is invariant under diffeomorphisms for any choice of the invariant tensor $g_{a b c}$.

One could also expect the presence of another first-class constraint, namely, the generator of timelike diffeomorphisms. However, as we shall see below, this symmetry is not independent from the other ones and hence its generator is a combination of the first-class constraints $G_{a}$ and $H_{i}$.

We now examine whether the first-class constraints $G_{a}$ and $H_{i}$ are independent and constitute a complete set. This depends on the properties of the invariant tensor $g_{a b c}$ and, for a definite choice of $g_{a b c}$, it also depends on the phase space location of the system. This is due to the fact that the constraint surface of the Chern-Simons theory is stratified into phase space regions where the matrix $\Omega_{a b}^{i j}$ has different ranks.

We will say that an invariant tensor $g_{a b c}$ is generic if and only if it satisfies the following condition: There exist solutions $F_{i j}^{a}$ of the constraints $K_{a}=0$ such that (i) the matrix $F_{k j}^{b}$ (with $b, j$ as row index and $k$ as column index) has maximum rank 4 , so that the only solution of $\xi^{k} F_{k j}^{b}=0$ is $\xi^{k}=0$ and therefore the four null eigenvectors $\left(v_{k}\right)_{j}^{b}=F_{k j}^{b}$, $(k=1, \ldots, 4)$ are linearly independent, and (ii) the $(4 N) \times(4 N)$ matrix $\Omega_{a b}^{i j}$ has the maximum rank compatible with (i), namely, $4 N-4$; in other words, it has no other null eigenvectors besides $\left(v_{k}\right)_{j}^{b}=F_{k j}^{b},(k=1, \ldots, 4)$.

We will also say that the solutions $F_{i j}^{a}$ of the constraints $K_{a}=0$ that allow for this condition to be satisfied are $g e$ neric. The reason for this name comes from the following observation. For a given generic $g_{a b c}$, a solution satisfying both conditions (i) and (ii) will still satisfy them upon small perturbations since maximum rank conditions correspond to inequalities and define open regions. Conversely, a solution not satisfying conditions (i) or (ii), i.e., located on the surface where lower ranks are achieved (defined by equations expressing that some nontrivial determinants vanish), will fail to remain on that surface upon generic perturbations consistent with the constraints. Nongeneric solutions of the constraint equations are also of physical interest but will not be considered here (see Ref. [10] for a more complete analysis).

The genericality condition represents the general case in the sense that it defines an open region in the space of the invariant tensors. Indeed, as we have pointed out, these al- gebraic conditions enforce inequalities. Therefore, to achieve a lower rank, some extra conditions would have to be satisfied and this would put $g_{a b c}$ on a surface of lower dimensionality in the space of the invariant tensors.

The physical meaning of the above algebraic conditions is straightforward. They simply express that the gauge transformations (2) and the spatial diffeomorphisms (3) are independent and that there are no other first-class constraints among the $\phi_{a}^{j}$ besides $H_{i}$.

In order to illustrate these points and to show that the genericality condition is not self-contradictory and can be actually satisfied, let us work out a simple example. Take a nondiagonal $g_{a b c}$ of the form

$$
g_{a 11}=0, \quad g_{a^{\prime} b^{\prime} 1} \equiv g_{a^{\prime} b^{\prime}}, \quad \text { invertible, }
$$

where $a^{\prime}, b^{\prime}, \ldots=2,3, \ldots, N$. Then, the constraints $K_{a}=0$ are solved by taking $F_{i j}^{a^{\prime}}=0$ and $F_{i j}^{1}$ arbitrary. The matrix $\Omega_{a b}^{i j}$ has the tensor product form

$$
\Omega_{1 a}^{i j}=0, \quad \Omega_{a^{\prime} b^{\prime}}^{i j}=g_{a^{\prime} b^{\prime}} \epsilon^{i j k l} F_{k l}^{1},
$$

and is thus of rank $4(N-1)$ provided that $F_{i j}^{1}$ is taken to be invertible. The invertibility of $F_{i j}^{1}$ also ensures that the only solution of $\xi^{k} F_{k j}^{b}=0$ is $\xi^{k}=0$. Therefore, we can conclude that the invariant tensor $g_{a b c}$ given in Eq. (17) is generic. Also, this example shows the stratification of phase space. While the solution that we have discussed [with $\operatorname{det}\left(F_{i j}^{1}\right)$ $\neq 0]$ is generic, solutions of the same form but with $\operatorname{det}\left(F_{i j}^{1}\right)=0$ belong to one of these lower dimensional nongeneric phase space regions.

Thus, for generic theories, the only first-class constraints are $G_{a}=0$ and $H_{i}=0$, which shows that the generator of timelike diffeomorphisms is not independent from $G_{a}$ and $H_{i}$. This may be verified explicitly by writing the action of a timelike diffeomorphism parametrized by $\xi^{\mu}=\left(\xi^{0}, 0\right)$ on $A_{i}^{a}$ as [see Eq. (3)],

$$
\delta_{\xi} A_{i}^{a}=\xi^{0} F_{i 0}^{a} .
$$

Now, the equations of motion (8) are $\Omega_{a b}^{i j} F_{0 j}^{b}=0$. Since the only zero eigenvectors of the matrix $\Omega_{a b}^{i j}$ are $F_{k j}^{b}$, there must exist some $\zeta^{k}$ such that $F_{j 0}^{b}=\zeta^{k} F_{j k}^{b}$. Inserting this result in Eq. (19) we obtain

$$
\delta_{\xi} A_{i}^{a}=\xi^{0} \zeta^{k} F_{i k}^{a},
$$

which is an improved spatial diffeomorphism with parameter $\xi^{0} \zeta^{k}$.

We can now count the number of local degrees of freedom in the generic case. We have, $2 \times 4 N$ canonical variables $\left(A_{i}^{a}, p_{a}^{i}\right), N$ first-class constraints $G_{a}$ associated with the gauge invariance, four first-class constraints $H_{i}$ associated with the (spatial) diffeomorphism invariance, and $4 N-4$ second-class constraints (the remaining $\phi_{a}^{i}$ ). Hence, we have

$$
\frac{1}{2}[8 N-2(N+4)-(4 N-4)]=2 N-2-N
$$

local degrees of freedom. The formula does not apply to $N=1$ because the spatial diffeomorphisms are not indepen- 
dent in that case, as can be checked directly on the canonical form $F=d x^{1} \wedge d x^{2}$. From (21) we see that, for $N=2$, there are no degrees of freedom. This happens because one does not use all the diffeomorphism invariance to bring the first $F^{1}$ to a canonical form. One may then use the residual diffeomorphism invariance to bring the second field strength $F^{2}$ to a canonical form also. However, for $N>2$, there are degrees of freedom.

The analysis has been performed so far in the Abelian case. In the non-Abelian case, the analysis proceeds similarly. The above formulas have been actually written in such a way that they remain true in the non-Abelian case. The only difference is that the invariance condition now strongly restricts the possible $g_{a b c}$. So one may fear that there could be a conflict between the invariance condition and the genericality condition. This is not the case and we have verified explicitly that the three-index invariant tensor of $\mathrm{SU}(p)$ $(2<p \leqslant 6)$ is generic. Likewise the gravitational ChernSimons theories in five dimensions are also generic and therefore do carry local degrees of freedom (this was anticipated in quite a different way by Chamseddine who analyzed perturbations around a nontrivial background [2]).

What has been done in five dimensions can be repeated in higher (odd) dimensions. Provided the invariant tensor $g_{a_{1} \cdots a_{n+1}}$ satisfies a genericality condition that is the straightforward generalization of the one appropriate to five dimensions, one finds that the canonical formulation of Chern-Simons theory involves $N+2 n$ first-class constraints and $2 n N-2 n$ second-class constraints in the generic case. The first-class constraints generate the gauge symmetries (2) and the spatial diffeomorphisms (3). As in five dimensions, the timelike diffeomorphisms can be expressed in terms of the other gauge symmetries. Since there are $2 n N$ conjugate pairs, the number of local degrees of freedom is equal to

$$
\text { No. }=n N-n-N \text {, }
$$

where $N>1$ and $n>1$. This expression vanishes only for $n=2$ and $N=2$. Again, one may also verify that the genericality condition is not self-contradictory by exhibiting invariant tensors that satisfy it. For instance, one may take a direct generalization of Eq. (17). The complete analysis, where the explicit isolation of the second-class constraints is performed and the Dirac brackets is computed, will be reported elsewhere [10].

When the invariant tensor $g_{a_{1} \cdots a_{n+1}}$ is not generic, $\Omega_{a b}^{i j}$ has further zero eigenvalues and thus, there are further gauge symmetries. This implies that the number of degrees of freedom is smaller than in the generic case and may even vanish. As we mentioned above, an extreme example is given by $N$ uncoupled Abelian gauge fields, where the extra gauge symmetries are diffeomorphisms acting independently on each individual copy.

To conclude, we have shown that higher dimensional Chern-Simons theories, even though constructed along the same topological pattern as in $2+1$ dimensions, do have local degrees of freedom provided that the invariant tensor that enters the action satisfies an appropriate genericality condition. This condition implies that there are no accidental gauge symmetries, so that the number of gauge symmetries grows more slowly with the dimension of the gauge group than the number of dynamical variables. This result cannot be anticipated by analyzing the case of a single Abelian field, which is not representative of the general case.

We are grateful to Claudio Teitelboim and Jorge Zanelli for useful conversations. M.B. was partially supported by Grants Nos. 1930910-93 and 1940203-94 from FONDECYT (Chile), a Grant from Fundación Andes (Chile), and by institutional support to the Centro de Estudios Científicos de Santiago provided by SAREC (Sweden) and a group of Chilean private companies (COPEC,CMPC,ENERSIS). L.J.G. was supported by the Ministerio de Educación y Ciencia (Spain) and the British Council. This work was supported in part by Research Funds from the European Community and by a Research Grant from F.N.R.S.
[1] R. Jackiw, Phys. Rev. Lett. 41, 1635 (1978).

[2] A. H. Chamseddine, Nucl. Phys. B346, 213 (1990); Phys. Lett. B 233, 291 (1989)

[3] A. Achúcarro and P. Townsend, Phys. Lett. B 180, 89 (1986); E. Witten, Nucl. Phys. B311, 46 (1998).

[4] D. Lovelock, J. Math. Phys. 12, 498 (1971).

[5] M. Bañados, C. Teitelboim, and J. Zanelli, Phys. Rev. D 49, 975 (1994).

[6] M. Bañados, C. Teitelboim, and J. Zanelli, Phys. Rev. Lett. 69, 1849 (1992); M. Bañados, M. Henneaux, C. Teitelboim, and J.
Zanelli, Phys. Rev. D 48, 1506 (1993).

[7] R. Floreanini and R. Percacci, Phys. Lett. B 224, 291 (1989).

[8] G. Barnich, M. Henneaux, and C. Schomblond, Phys. Rev. D 44, R939 (1991).

[9] See, e.g., M. Henneaux and C. Teitelboim, Quantization of Gauge Systems (Princeton University Press, Princeton, New Jersey, 1992).

[10] M. Bañados, L. J. Garay, and M. Henneaux, The Dynamical Structure of Higher Dimensional Pure Chern-Simons Theories (unpublished). 